\title{
NON-UNIVERSAL PAIRING SYMMETRY AND PSEUDOGAP PHENOMENA IN HOLE- AND ELECTRON-DOPED CUPRATE SUPERCONDUCTORS
}

\author{
N.-C. YEH* and C.-T. CHEN \\ Department of Physics, California Institute of Technology \\ Pasadena, CA 91125, USA
}

\begin{abstract}
Experimental studies of the pairing state of cuprate superconductors reveal asymmetric behaviors of the hole-doped (p-type) and electron-doped (n-type) cuprates. The pairing symmetry, pseudogap phenomenon, low-energy spin excitations and the spatial homogeneity of the superconducting order parameter appear to be non-universal among the cuprates, which may be attributed to competing orders. We propose that the nonuniversal pseudogap and nano-scale variations in the quasiparticle spectra may be the result of a charge nematic $(\mathrm{CN})$ phase stabilized by disorder in highly two-dimensional (2D) p-type cuprates. The CN phase is accompanied by gapped spin excitations and competes with superconductivity (SC). In contrast, gapless spin excitations may be responsible for the absence of pseudogap and the presence of excess sub-gap spectral weight in the momentum-independent quasiparticle spectra of n-type cuprates. The physical implications and further verifications for these conjectures are discussed.
\end{abstract}

Keywords: Cuprate superconductors; pairing symmetry; pseudogap; spin excitations.

\section{Introduction}

Recent spectroscopic studies of various hole-doped (p-type) and electron-doped (ntype) cuprate superconductors have revealed that some phenomena widely perceived as essential for the occurrence of cuprate superconductivity are in fact not universal, and that substantial asymmetries exist between p-type and n-type cuprates. ${ }^{1,2,3,4}$ More specifically, the pairing symmetry in p-type cuprates appears to be predominantly $d_{x^{2}-y^{2}}$ or $\left(d_{x^{2}-y^{2}}+s\right)$ for all doping levels, both involving four nodes in the momentum $(k)$ space and $k$-dependent superconducting energy gap $\left(\Delta_{k}\right) .^{1,2,3}$ In contrast, quasiparticle tunneling spectra of infinite-layer and one-layer n-type cuprates studied to date are all $k$-independent and are therefore consistent with $s$ wave pairing, ${ }^{3,4,5}$ although experiments other than tunneling spectra suggest doping dependent pairing symmetry in the one-layer n-type cuprates. ${ }^{6,7,8}$ These different pairing symmetries have been attributed to competing energy scales in the cuprates. ${ }^{1}$ The response in the pairing state to quantum impurities that substitute $\mathrm{Cu}$ in the $\mathrm{CuO}_{2}$ plane is also asymmetric between p-type and n-type cuprates, the former being sensitive to both magnetic and non-magnetic impurities as expected

${ }^{*}$ Corresponding author. E-mail: ncyeh@caltech.edu 
for $d$-wave superconductors, ${ }^{2,3,9,10}$ while the latter being only sensitive to magnetic impurities as expected for typical $s$-wave superconductors. ${ }^{4}$ Moreover, scanning tunneling and angular resolved photoemission spectroscopic (STS and ARPES) studies $^{11,12}$ reveal pseudogap features well above the superconducting transition $\mathrm{T}_{c}$ in optimal and underdoped $\mathrm{Bi}_{2} \mathrm{Sr}_{2} \mathrm{CaCu}_{2} \mathrm{O}_{8+\mathrm{x}}$ (Bi-2212) single crystals, and these pseudogap features evolve smoothly below $T_{c}$ into the so-called "dip-hump" satellite features at energies above the superconducting gap. In contrast, no quasiparticle pseudogap has been identified in $\mathrm{YBa}_{2} \mathrm{Cu}_{3} \mathrm{O}_{7-\delta}(\mathrm{YBCO})$ above $T_{c},{ }^{13}$ although satellite features similar to those of Bi-2212 also exist at $T<T_{c} .{ }^{2,3,13}$ On the other hand, neither spectral satellite features below $T_{c}$ nor quasiparticle pseudogap above $T_{c}$ have ever been reported for the n-type cuprates. ${ }^{4,15,16}$ The low-energy spin excitations inferred from neutron scattering studies also reveal substantial differences between the p-type and n-type cuprates, the former being associated with gapped spin excitations ${ }^{17}$ while the latter being gapless. ${ }^{18,19}$ Finally, nano-scale spatial variations in the tunneling gap of under- and optimally doped Bi- $2212^{20}$ differ from the spatially homogeneous pairing potential in YBCO and n-type cuprates according to $\mathrm{STS}^{2,3,4}$ and nuclear magnetic resonance (NMR) studies. ${ }^{21}$

The aforementioned non-universal phenomena strongly suggest that the asymmetric behaviors between n-type and p-type cuprates cannot be easily reconciled with a one-band Hubbard model that asserts particle-hole symmetry, ${ }^{1}$ and that better understanding of the pairing mechanism relies on determining the physical origin of these non-universal phenomena and identifying the ubiquitous features among all cuprates. In the following, we propose a scenario that provides feasible account for the non-universal pseudogap phenomenon, satellite features and spatial homogeneity of the pairing potential. The physical implications of and more stringent tests for this scenario are discussed.

\section{Pseudogap Phenomena, Spin Excitations and Spatial Homogeneity of the Order Parameter}

The absence of pseudogap in n-type cuprates ${ }^{4,15,16}$ implies that the pseudogap phenomenon cannot be a prerequisite for cuprate superconductivity. To better understand the physical origin of the pseudogap, we compare the quasiparticle tunneling spectra in under- and optimally doped Bi-2212 with those of YBCO and n-type cuprates, as exemplified in Figs. 1(a)-(b). In the case of Bi-2212, a typical c-axis tunneling spectrum with sharp superconducting coherence peaks at $T<T_{c}$ is often accompanied by the dip-hump satellite features at higher energies, ${ }^{11,20}$ and the superconducting coherence peaks diminish with increasing temperature. ${ }^{11}$ The other types of spectra in Bi-2212, which typically occur at locations only a few nanometers away from regions showing the first type of spectra ${ }^{20}$ reveal rounded peaks and non-vanishing density of states at zero bias, as exemplified in the inset of Fig. 1 (a), and these rounded features generally persist well above $T_{c} \cdot{ }^{11}$ In contrast, only one type of c-axis tunneling spectra with both sharp coherence peaks and satellite 
features can be observed in $\mathrm{YBCO},{ }^{1,2,3,13,14}$ as shown in the main panel of Fig. 1(a), and all interesting features completely vanish above $T_{c} \cdot{ }^{13}$
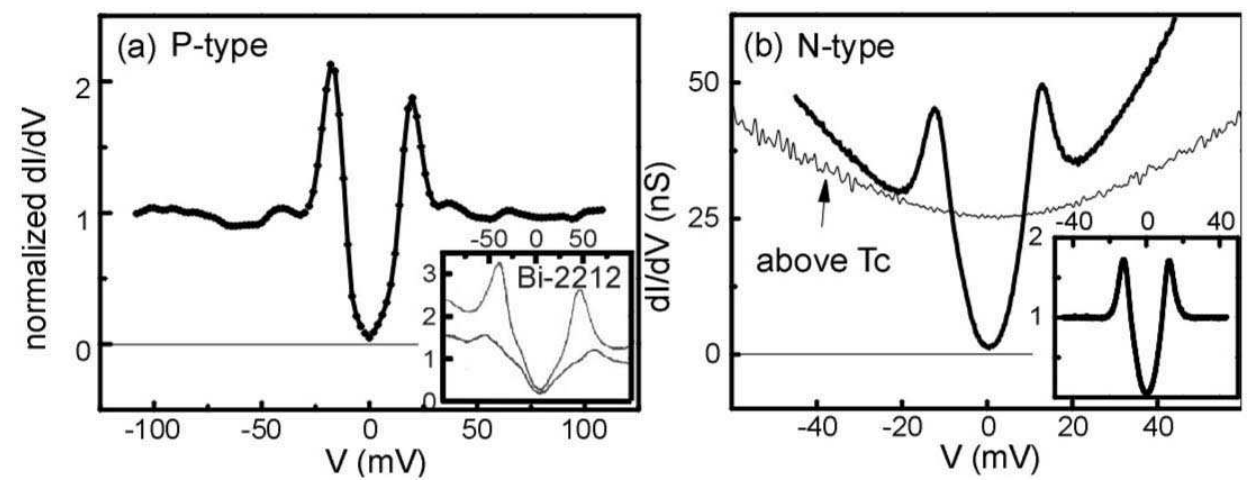

Fig. 1. Comparison of the quasiparticle tunneling spectra between p-type and n-type cuprates. (a) c-axis tunneling spectrum of an optimally doped YBCO at $4.2 \mathrm{~K} .{ }^{2,3}$ The inset shows sketches of two typical types of c-axis tunneling spectra of a slightly underdoped Bi-2212.11,20 (b) Momentumindependent tunneling spectra of an infinite-layer n-type cuprate $\mathrm{Sr}_{0.9} \mathrm{La}_{0.1} \mathrm{CuO}_{2}$ at $4.2 \mathrm{~K} \ll T_{c}=$ $43 \mathrm{~K}$ and above $T_{c}{ }^{3,4}$ The inset shows the normalized spectrum of the main panel.

The phenomena described above may be understood in terms of a "pseudogap phase" competing with superconductivity as follows. Theoretical investigations ${ }^{22,23}$ have shown that two competing orders $\mathrm{A}$ and $\mathrm{B}$ of a system generally result in three types of phase diagrams as a function of the chemical potential $\mu$, as illustrated in Figs. 2(a)-(c). For the phase diagram depicted in Fig. 2(a), nano-scale phase separations of $\mathrm{A}$ and $\mathrm{B}$ are expected if the chemical potential coincides with the critical value $\mu_{c}$. Noting that the same chemical potential can be associated with different doping levels, we suggest that the under- and optimally doped Bi-2212 may belong to this category. On the other hand, long-range spatial homogeneity of the order parameter is expected for the coexisting phase A/B depicted in Fig. 2(b). This scenario may correspond to the observed spatial homogeneity of the quasiparticle spectra in the pairing state of $\mathrm{YBCO}$, with coexisting superconducting coherence peaks and satellite features. Moreover, it could account for the absence of quasiparticle pseudogap above $T_{c}{ }^{13}$ if the condition $\mu_{x} \leq \mu \leq \mu_{b}$ is satisfied.

Concerning the microscopic makeup of the pseudogap phase, we note that according to the Mermin-Wagner theorem, ${ }^{24}$ no long-range order with spontaneous breaking of continuous symmetry can exist in a strictly 2D system. Given that superconductivity involves spontaneous $\mathrm{U}(1)$ symmetry-breaking below $T_{c}$, one would expect the absence of long-range superconducting order in highly $2 \mathrm{D}$ systems such as Bi-2212. Meanwhile, if a second phase relatively close in energy to superconductivity (SC) exists and if it can be stabilized by disorder, phase separations at between the second phase and SC at relatively small spatial scales would become 


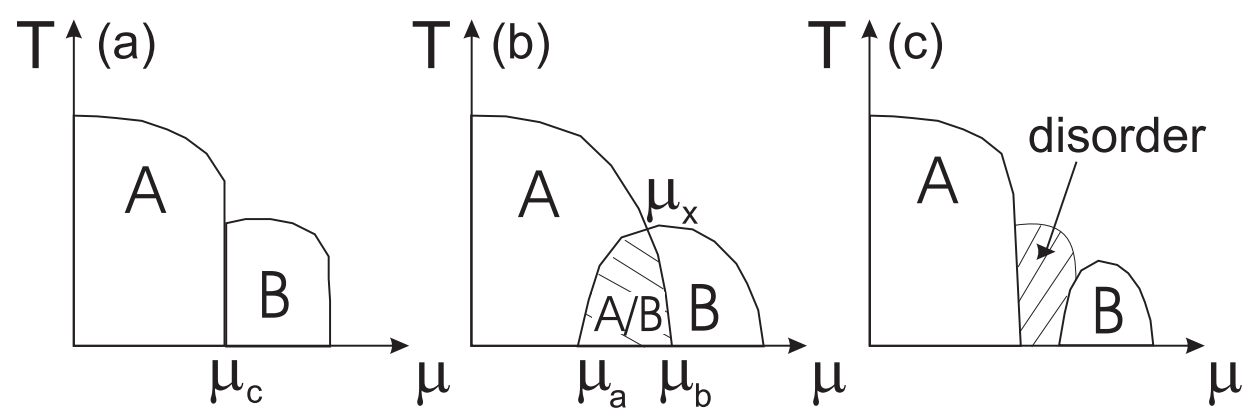

Fig. 2. A schematic illustration of three possible temperature $(T)$ vs. chemical potential $(\mu)$ phase diagrams associated with two competing orders $\mathrm{A}$ and $\mathrm{B}$, depending on details of the Hamiltonian. $^{22,23}$ (a) A and $\mathrm{B}$ are separated by a first-order critical line or critical point. (b) $\mathrm{A}$ and $\mathrm{B}$ coexist over a finite range of chemical potential, and the coexisting phase A/B is separated from A and B with second-order critical lines. (c) A and B are intervened by disorder. In p-type cuprates, A may be a charge nematic phase associated with gapped spin excitations, and B may correspond to the superconducting phase.

highly probable. We suggest that the pseudogap phase may be a "charge nematic" $(\mathrm{CN})$ phase which involves localized stripe-like charge modulations. The CN phase is energetically more favorable than charge stripes because of its reduced Coulomb repulsion relative to the latter. Moreover, CN phase could fulfill the role of accommodating incommensurate hole doping via local spin fluctuations, which is consistent with neutron scattering experiments that attribute the observation of gapped spin excitations to charge modulations in p-type cuprates. ${ }^{17}$ Under this premise, the phase boundary between $\mathrm{CN}$ and $\mathrm{SC}$ would involve defected spin configurations because SC is effectively a spin liquid, and the defected spin configurations in $2 \mathrm{D}$ could be either positive or negative in sense, similar to the disclinations or vortex/anti-vortex pairs in 2D. Moreover, these locally defected configurations could be statically pinned by disorder, analogous to the observed quantum Hall nematic $(\mathrm{QHN})$ phase in 2D electron gas (2DEG). ${ }^{25}$ On the other hand, introduction of 3D coupling not only stabilizes long-range SC order but also permits smooth relaxation of defected spin configurations, thereby eliminating the need for phase separation. Thus, our conjecture of CN being the pseudogap phase competing with SC can account for the long-range spatial homogeneity of the pairing potential in 3D YBCO and the nano-scale phase separations in $2 \mathrm{D} \mathrm{Bi}-2212$ for $T<T_{c}$. In contrast, gapless spin excitations in n-type cuprates ${ }^{18,19}$ are unlikely related to local charge modulations. Therefore there is no obvious competing order with SC near the optimal doping of n-type cuprates, which explains the long-range homogeneity of the pairing potential and the absence of pseudogap in zero field. ${ }^{1,4,15,16}$ 


\section{Spectroscopic Evidence for Gapless Spin Excitations in N-Type Cuprates}

In the case of n-type cuprates, while the momentum-independent quasiparticle tunneling spectra and the insensitivity of superconductivity to non-magnetic impurities are strongly supportive of s-wave pairing symmetry, ${ }^{4,26}$ the quasiparticle spectra differ substantially from the BCS prediction for conventional s-wave superconductors. ${ }^{4}$ As illustrated in Fig. 3, we note that there are excess sub-gap quasiparticle density of states (DOS), which cannot be accounted for by either disorder effects ${ }^{4}$ or anisotropy in the fully gapped Fermi surface. ${ }^{26}$ Recall that neutron scattering experiments reveal gapless spin excitations in n-type cuprates, ${ }^{18,19}$ we conjecture that the excess sub-gap quasiparticle DOS is the result of quasiparticle coupling to the background gapless spin excitations. However, theoretically it is not possible to have gapless spin excitations above one dimension without the spontaneous breaking of a continuous symmetry. ${ }^{24}$ In the absence of spontaneous $\mathrm{SU}(2)$ symmetry-breaking in the superconducting state, the only possibility for generating gapless spin excitations in n-type cuprates is to invoke non-trivial coupling of spin fluctuations to the phase of the superconducting order parameter. The resulting phase fluctuations have been shown to yield broadening of the quasiparticle spectra, ${ }^{27}$ which is consistent with our experimental finding. ${ }^{4}$ Such coupling can also justify the existence of gapless spin excitations through spontaneous breaking of the U(1) symmetry-breaking in the superconducting state. However, a rigorous proof from first principle will require deriving an effective perturbation Hamiltonian $\mathcal{H}_{1}$ from the coupling of the gapless spin excitations to the phase of the superconducting order parameter. Once $\mathcal{H}_{1}$ is known, one can obtain the Green's function $\mathcal{G}$ of the perturbed system, and the quasiparticle DOS $\mathcal{N}(E)$ can be derived through the imaginary part of $\mathcal{G}$ via the relation $\mathcal{N}(E)=-\operatorname{Im}[\mathcal{G}] / \pi$. This theoretical issue awaits further investigation.

\section{Discussion}

We have proposed in the previous sections that different low-energy spin excitations in p-type and n-type cuprate superconductors are responsible for various nonuniversal characteristics such as the pseudogap phenomenon and satellite features in the quasiparticle spectra. We further suggest that a charge nematic $(\mathrm{CN})$ phase as a competing order with superconductivity can gives rise to nano-scale spectral variations in highly $2 \mathrm{D}$ p-type cuprates while yielding long-range spectral homogeneity in 3D p-type cuprates. The conjecture of a competing CN phase is feasible for a number of reasons. First, $\mathrm{CN}$ is much closer in energy to $\mathrm{SC}$ than other known competing orders such as antiferromagnetism and stripes. Moreover, the association of the CN phase with gapped spin excitations is consistent with the doping dependence of pseudogap in p-type cuprates, because the spin stiffness and therefore the energy required for gapped spin excitations decreases with increasing hole doping. In addition, $\mathrm{CN}$ as a near-by competing order would be consistent with the

"checker-board" short-range charge modulations observed within the vortex core of 


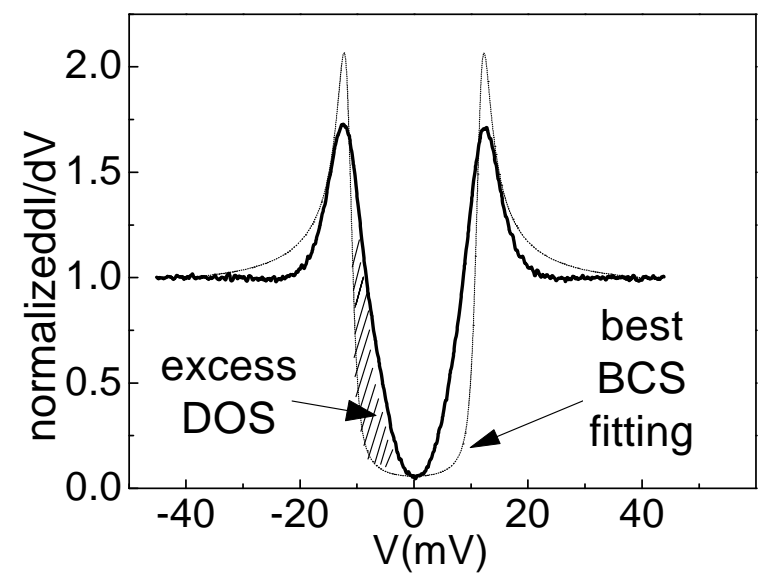

Fig. 3. Comparison of the normalize quasiparticle tunneling spectrum of the infinite-layer n-type cuprate $\mathrm{Sr}_{0.9} \mathrm{La}_{0.1} \mathrm{CuO}_{2}$ with the best BCS fitting at $4.2 \mathrm{~K}$, showing apparent disagreement and excess sub-gap quasiparticle DOS relative to conventional BCS theory. ${ }^{4}$ We attribute the excess sub-gap DOS to quasiparticles damping by the gapless spin excitations in the system.

Bi-2212 28,29 upon the suppression of SC inside the vortex core by magnetic fields. Finally, the absence of gapped spin excitations in n-type cuprates implies absence of a competing $\mathrm{CN}$ phase, which can be reconciled with the long-range spectral homogeneity in n-type cuprate as well as the absence of pseudogap above $T_{c}$ and missing satellite features below $T_{c}$.

Despite the consistencies, a number of issues still await further verifications. First, rigorous theoretical proof for the stability of a ground state CN phase and its dependence on the doping level and disorder will be necessary. In addition, the evolution of the CN phase with temperature, the spatial distribution of the CN and SC phase boundaries, and the effect of magnetic fields are issues to be addressed both empirically and theoretically. Finally, the microscopic coupling mechanism of the gapless spin excitations in n-type cuprates to phase fluctuations of the order parameter must be resolved.

\section{Summary}

We have reviewed asymmetric behaviors among p-type and n-type cuprates and discussed possible physical origin for various non-universal phenomena. In the case of p-type cuprates, we propose a charge nematic $(\mathrm{CN})$ phase, which is associated with gapped spin excitations, as a competing order to superconductivity (SC). The competition of $\mathrm{CN}$ and $\mathrm{SC}$ can result in nano-scale phase separation in 2D while yielding homogeneous order parameter in 3D. On the other hand, we suggest that the gapless spin excitations in n-type cuprates may be responsible for the excess sub-gap quasiparticle density of states and the missing satellite features below $T_{c}$, as well as the absence of pseudogap above $T_{c}$. These considerations of empirical facts 
suggest that spin correlation plays an essential role in cuprate superconductivity.

\section{Acknowledgments}

This work is supported by the National Science Foundation through Grant \#DMR0103045 .

\section{References}

1. N.-C. Yeh, in Bulletin of Assoc. Asia Pacific Phys. Soc. 12, 2 (2002); and references therein.

2. N.-C. Yeh et al., Phys. Rev. Lett., 87, 087003 (2001).

3. N.-C. Yeh et al., Physica C 367, 174 (2002).

4. C.-T. Chen et al., Phys. Rev. Lett. 88, 227002 (2002).

5. L. Alff et al., Phys. Rev. Lett. 83, 2644 (1999).

6. C. C. Tsuei and J. Kirtley, Phys. Rev. Lett. 85, 182 (2000).

7. J. A. Skinta et al., Phys. Rev. Lett. 88, 207005 (2002).

8. A. Biswas et al., Phys. Rev. Lett. 88, 207004 (2002).

9. S. H. Pan et al., Nature 403, 746 (2000).

10. E. W. Hudson et al., Nature 411, 920 (2001).

11. Ch. Renner et al., Phys. Rev. Lett. 80, 149 (1998).

12. Z. X. Shen and D. S. Desau, Phys. Rep. 253, 1 (1995).

13. I. Maggio-Aprile et al., J. Electron Spectrosc. 109, 147 (2000).

14. J. Y. T. Wei et al., Phys. Rev. Lett. 81, 2542 (1998).

15. S. Kleefisch et al., Phys. Rev. B 63, 100507 (2001).

16. G. V. M. Williams et al., Phys. Rev. B 65, 224520 (2002).

17. M. A. Kastner et al., Rev. Mod. Phys. 70, 897 (1998); and references therein.

18. K. Yamada et al., J. Phys. Chem. Solids 60, 1025 (1999).

19. T. Takahashi et al., J. Phys. Chem. Solids 62, 1025 (2001).

20. S. H. Pan et al., Nature 413, 282 (2001).

21. J. Bobroff et al., Phys. Rev. Lett. 89, 157002 (2002).

22. S.-C. Zhang, Science 275, 1089 (1997).

23. J. Burgy et al., Phys. Rev. Lett. 87, 277202 (2001).

24. See, for example, E. Frandkin, Field Theory of Condensed Matter Systems, AddisonWesley (1991).

25. G. Finkelstein et al. Science 289, 90 (2000).

26. N.-C. Yeh et al., J. Low Temp. Phys., (2002) (in press); cond-mat/0207594.

27. H.-J. Kwon and A. T. Dorsey, Phys. Rev. B 59, 6438 (1999).

28. B. Lake et al., Science 291, 1759 (2001).

29. J. E. Hoffman et al., Science 295, 466 (2002). 